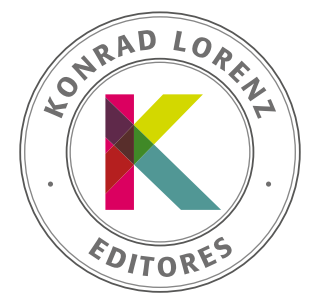

\title{
Propiedades psicométricas de la Utrecht Work Engagement Scale en estudiantes de educación
}

\author{
Javier Cachón-Zagalaza , Amador Lara-Sáncheza , María Luisa Zagalaz-Sánchez ${ }^{a}$, \\ Inés López-Manrique ${ }^{\mathrm{b}}$ y Carmen González González de Mesa ${ }^{\mathrm{b}}$
}

a Universidad de Jaén, España
${ }^{b}$ Universidad de Oviedo, España

Recibido el 28 de octubre de 2017; aceptado el 23 de febrero de 2018

\author{
PALABRAS CLAVE \\ Engagement \\ académico, \\ UWES-S, \\ compromiso \\ académico, \\ análisis factorial, \\ estudiantes \\ universitarios \\ de educación
}

\begin{abstract}
Resumen Este trabajo tiene por objeto aplicar la escala UWES-S a los sujetos que se están formando como profesores y estudiar la inferencia de las variables sociodemográficas y personales en el compromiso con sus estudios. Material y Método: Para esta investigación, instrumental y empírica, se ha utilizado la versión española del Utrecht Work Engagement Scale (UWES-S), adaptada a dichos estudios, que evalúa el grado de compromiso individual (motivación) del sujeto hacia la actividad que realiza. El cuestionario comprende 17 ítems, con escala Likert de cinco posiciones. Se han usado cuatro programas estadísticos: SPSS.22, Factor.10, MPlus.7 y G*Pwer 3.1.9.2. Han participado 373 estudiantes del grado de Maestro ( $n=196$ de Educación Primaria, $n=85$ de Educación Infantil) y $n=92$ del Máster de Formación del Profesorado de Secundaria) de dos universidades del norte y sur de España, y una universidad de Chile. Tras el análisis de resultados y la discusión, se concluye que existen diferencias significativas en cuanto al sexo y situación geográfica de las universidades. Las mujeres se sienten más comprometidas con sus estudios, así como los estudiantes del sur de España y los latinoamericanos, al compararlos con los del norte.

(c) 2018 Fundación Universitaria Konrad Lorenz. Este es un artículo Open Access bajo la licencia CC BY-NC-ND (http://creativecommons.org/licenses/bync-nd/4.0/).
\end{abstract}

* Autor para correspondencia.

Correo electrónico: Izagalaz@ujaen.es

http://dx.doi.org/10.14349/sumapsi.2018.v25.n2.3

0120-0534/@ 2018 Fundación Universitaria Konrad Lorenz. Este es un artículo Open Access bajo la licencia CC BY-NC-ND (http://creativecommons.org/licenses/by-nc-nd/4.0/). 


\section{KEYWORDS}

Academic

engagement, UWES-S, academic commitment, factor analysis, university education students
Psychometric Properties of the Utrecht Work Engagement Scale in Teacher Training Students

\begin{abstract}
This paper undertook to apply the UWES-S scale to the individuals training as teachers, and to study the inference of sociodemographic and personal variables with their commitment in their studies. Material and method: This is an instrumental and empirical research wherein we utilized the Spanish version of the Utrecht Work Engagement Scale-UWES-S, which evaluates the degree of individual commitment inherent in the activity performed. This survey contains 17 items under a Likert scale of 5 positions. We utilized three statistical software programs: SPSS.22, Factor.10, MPlus.7, and G*Pwer 3.1.9.2. 373 Education Undergraduate students ( $n=196$ of Primary, $n=85$ of Preschool) and $n=92$ students under the Master Degree in Secondary Education partook in this study. Say students appertained to two Universities of north and south of Spain, and a University of Chile. After analyzing the results and the discussion, we have concluded that there are significant differences in the personal variables, both in the social demographical character and the geographical location of the Universities. In addition, the female students, and students from southern Spain and Latin Ameri$\mathrm{ca}$, are more committed to their studies as compared to students from the North of Spain. (c) 2018 Fundación Universitaria Konrad Lorenz. This is an open access article under the CC BY-NCND license (http://creativecommons.org/licenses/bync-nd/4.0/).
\end{abstract}

El Engagement o Compromiso académico, es un constructo teóricamente trifactorial (vigor, absorción y dedicación), actualmente medido a través del cuestionario UWES-S (Schaufeli \& Bakker, 2003) cuyas propiedades psicométricas se han estudiado en Europa, con lo que se confirma la estructura de dichos tres factores (Parra \& Pérez, 2010).

El engagement en el trabajo se asume como el polo opuesto al burnout. Al contrario de los que padecen burnout, los empleados con engagement tienen un sentido de conexión energética y afectiva con su trabajo y se perciben con las capacidades suficientes para afrontar las demandas de su actividad. Maslach y Leiter (1997), asumen que engagement y burnout son polos opuestos, el primero positivo y el segundo negativo. Definen el burnout como agotamiento, cinismo y baja eficacia profesional, mientras el engagement se caracteriza por energía, involucración y eficacia.

Para entender el compromiso académico desde la óptica de los estudiantes de educación se plantean brevemente en este apartado las características del profesorado en formación que impartirá docencia en las distintas etapas de la enseñanza.

El profesorado de Educación Infantil (El) trabaja con niños en edades comprendidas entre 0 y 6 años (enseñanza no obligatoria). Como en la mayor parte de los países europeos la El en España está articulada en dos ciclos: $1^{\circ}$ de 0 a 3 y $2^{\circ}$ de 3 a 6 años, dependiendo el $1^{\circ}$ ciclo, en la mayoría de los casos, de los Técnicos Superiores en El, que han cursado estudios de Formación Profesional de Grado Superior. El $2^{\circ}$ ciclo en todo el territorio nacional depende de las Consejerías de Educación, y es ahí donde los maestros especialistas en El desarrollarán su trabajo. La etapa de El, muy condicionada por la herencia y el contexto, es en la que más se respeta el equilibrio entre el desarrollo de las distintas capacidades del niño (física, emocional, cognitiva). Las maestras (la mayoría son mujeres) de El presentan un estilo profesional presidido por el optimismo y la motivación, pese a las condiciones a veces adversas (Paniagua, 2009).

El profesorado de Educación Primaria (EP) trabaja con niños de entre 6 a 12 años, cuyas características cognitivas, psicológicas, motrices y afectivas se desarrollan a lo largo de la etapa, variando desde el principio hasta el final de este periodo educativo, momento en que el alumnado consigue, por lo general, el estadio de operaciones formales. Ello supone cambios decisivos en la forma de aprender y relacionarse, y consiste en alcanzar el pensamiento abstracto (Zagalaz, Cachón, \& Lara, 2014). La motivación de los profesionales hacia esta etapa suele ser menor que para $\mathrm{El}$, siendo los cursos $3^{\circ}$ y $4^{\circ}$ los preferidos por los maestros.

El profesorado de Educación Secundaria (ES) se ocupa de los adolescentes, chicos y chicas en el "período de la vida humana que sigue a la niñez y precede a la juventud" (RAE, 2016) y en pleno cambio fisiológico y emocional (Mesana, 2013). Esta etapa que finaliza al llegar a la edad adulta, aproximadamente a los 18 años (OMS, 2004; Villarreal, Sánchez, \& Musitu, 2010), se corresponde con la ES, tanto obligatoria (ESO, 12-16 años) como opcional (Bachillerato y FP, 16-18 años).

Para todos los niveles, el compromiso del futuro profesorado con la realización de estos estudios y su desempeño profesional tiene un marcado carácter vocacional y conlleva la consideración del autoconcepto desde las cinco dimensiones que señalaron Shavelson, Hubner y Stanton (1976), académico, social, emocional, familiar y físico (que corroboran García \& Musitu, 2014). En ellas destaca la percepción del desempeño de su rol como estudiante y profesional; la impresión de su cometido; su estado emocional y grado de compromiso; la sensación de su integración en el medio familiar; y la apreciación de su aspecto.

Incidiendo en el estudio del engagement, López Alonso (2011), en su estudio sobre el ocio y tiempo libre de los universitarios, lo analiza como factor influyente en el tipo de enfoque que los alumnos tienen de su aprendizaje, la orientación que le dan y sus afectos positivos (compromiso). Los resultados coinciden con los de otros autores (Carrasco, Corte \& León, 2010; Lykken, 2000; Raigosa \& Marín, 2010; Salanova \& Schaufeli, 2004), quienes explican que las consecuencias del engagement, son las actitudes positivas que potencian la salud y el bienestar de las personas. En la misma línea se sitúan Gómez et al. (2015) en un estudio llevado a cabo en Chile para averiguar la relación entre el bienestar y el rendimiento académico en alumnos de primer año de medicina, así como López-Alonso, López-Aguado, Fernán- 
dez-Martínez, Liébana-Presa y Gutiérrez-Provecho (2016), en su trabajo con estudiantes de distintas titulaciones de la Universidad de León en España.

Otros abordan el concepto de engagement en el trabajo y en contextos educativos y su relación con el desempeño académico (Casuso-Holgado, 2011); algunos opinan que hay gran evidencia de que los factores de disposición estén relacionados con actitudes hacia el trabajo o engagement (Arraigada \& Musticchio, 2015).

Asimismo Martínez y Salanova (2003) analizan el burnout y el engagement en estudiantes universitarios para establecer relaciones con variables demográficas, desempeño y desarrollo profesional. Los resultados muestran diferencias significativas en función de la edad, el sexo y la titulación. También se señalan posibles predictores del desempeño y el desarrollo profesional.

Rosales (2014) investiga sobre el contraste de la imagen que un grupo de futuros maestros tiene de su profesión con la realidad de la misma. Los resultados revelan una importante sensibilización de los estudiantes de magisterio sobre sus expectativas de futuro y la realidad en el desarrollo de las mismas, es decir su compromiso.

Además existen otros factores que pueden condicionar el compromiso de los futuros docentes con sus estudios, tales como la resiliencia o capacidad de adaptación al estrés, que puede afectar la motivación y el comportamiento de los estudiantes. La angustia psicológica, analizada por Chen et al. (2016) con personal sanitario que exploran el efecto de resistencia emocional a ese trabajo que podría trasladarse al contexto educativo, especialmente en los primeros años de docencia.

Asimismo entre las características de los futuros docentes y su compromiso con sus estudios aparece la competencia emocional o capacidad para percibir, expresar, comprender, regular y controlar emociones (propias y ajenas), por tanto de vital importancia en ese desempeño.

Para finalizar este apartado se hace referencia al instrumento usado en el estudio y su importancia. Se trata del Utrecht Work Engagement Scale (UWES, Escala de compromiso de trabajo de Utrecht; UWES-S, versión para estudiantes), escala de la Universidad de Utrech, utilizada en diferentes ámbitos (sanitarios en Chile, trabajadores de distintos sectores en Puerro Rico, Cuba y Argentina, deportistas y fisioterapeutas en España, así como en diferentes estudios universitarios, tanto en Latinoamérica como en España.

El UWES pregunta directamente por la situación motivacional del sujeto (laboral/estudiante). Silva, Carena y Canuto (2013), lo usaron para medir el voluntariado de los estudiantes universitarios. También Da Silva, León, Perea y Bermejo (2014) calcularon la relación entre convencionalidad social y engagement en esos estudiantes. Lo mismo que Parra (2014) cuando insiste sobre la relación entre el nivel de engagement y el rendimiento académico teórico/práctico en las Facultades de Medicina.

Parra y Pérez (2010), analizan el compromiso académico de 164 estudiantes de psicología a través del cuestionario UWES-S (Schaufeli, Martínez, Marqués-Pinto, Salanova, \& Bakker, 2002) cuyas propiedades psicométricas se han estudiado en Europa, confirmándose dos factores emergentes: predisposición a estudiar y satisfacción con los estudios.

Con respecto a las variables personales, habitualmente las mujeres son mayoría en la profesión docente, el grupo de edad encuestado tiene para El y EP, entre 18-23 años y para ES, a partir de 24. Los estudios que realizan para EI y EP son las dos especialidades del grado de Maestro, y para ES cualquier grado previo a la realización del Máster de Profesorado. Se pueden cursar en todo el territorio nacional y en el extranjero, siempre que pasen por un proceso de convalidación si estudian fuera de la Unión Europea. Los estudios de Maestro se realizan en todas las Universidades públicas españolas y en muchas privadas. En este caso los sujetos participantes son estudiantes de universidades del norte o del sur de España y de Chile.

El objetivo de este estudio ha sido revalidar la escala UWES-S para estudiar la influencia y relación que pueden mantener las variables género, grupo de edades, tipo de estudios y situación geográfica de la universidad donde están matriculados los sujetos analizados, con los diferentes factores resultantes del compromiso con estudios de formación de profesorado de EI, EP y ES.

\section{Método}

\section{Muestra}

Han participado 373 estudiantes ( $n=196$ de EP, $n=85$ de El y $n=92$ del Máster de Profesorado de ES). El 25.2\% son varones y el $74.8 \%$ mujeres. Las edades oscilan entre 19 y 51 años, el $32.7 \%$ menos de 21 años, el $43.7 \%$ entre 21 y 24 y el $23.6 \%$ más de 24 años. En cuanto al curso en el que están matriculados, el $18 \%$ son de primero, el $30 \%$ de segundo, el $12 \%$ de tercero, el $15 \%$ de cuarto y el resto en el Máster en Profesorado de ESO, Bachillerato, Formación Profesional y Enseñanza de Idiomas. Se han seleccionado universitarios del sur de España (68\%), del norte de España (26.8\%) y de Latinoamérica (4\%).

\section{Instrumento}

El instrumento utilizado para su adaptación y aplicación ha sido la versión española del UWES-S de Schaufeli et al. (2002), validado para España por Belando, Ferriz-Morell, \& Moreno-Murcia (2012), para evaluar el grado de compromiso individual del deportista hacia el deporte que practica. Comprende 17 ítems y utiliza una escala Likert de cinco posiciones, que oscilan desde 1 = totalmente en desacuerdo hasta 5 = totalmente de acuerdo, con los ítems planteados. En el cuestionario se han incluido una serie de preguntas de tipo personal (sexo, edad, modalidad que cursan, universidad dónde cursan sus estudios, etc.) para profundizar y reforzar los resultados.

\section{Procedimiento}

Se ha obtenido el permiso del Comité Ético de las Universidades implicadas en este estudio y enviado un e-mail solicitando la colaboración a varios centros universitarios que forman a docentes de EI, EP y ES en España y en Chile. Conseguida la contestación favorable se ha enviado el enlace del cuestionario via e-mail a varios docentes de dichos centros para que los hicieran llagar a sus estudiantes. El cuestionario iba acompañado de una carta explicativa de los objetivos del estudio y solicitaba la colaboración y 
participación voluntaria. Se les advierte la importancia de la cumplimentación de todos los ítems y se garantiza el anonimato.

El programa de recogida de datos ha estado abierto durante un mes y a partir de este momento se procedió a cerrarlo para comenzar con el análisis de los resultados.

\section{Análisis de datos}

Los resultados de la investigación se muestran en dos partes. Primero, una investigación instrumental basada en la revalidación del cuestionario utilizado y, en segundo lugar, los resultados de la investigación empírica, donde se exponen los datos descriptivos de todas las variables y se dilucida la ascendencia que algunas variables tienen sobre otras y sobre los factores resultantes, así como las relaciones de determinadas variables independientes con los mismos.

Se ha tomado la decisión de realizar la investigación instrumental al comprobar que el cuestionario previsto había sido gestado fuera de España y aplicado, una vez traducido al castellano, fundamentalmente en países latinoamericanos, y aunque también se ha utilizado, y en algunos casos hecho la validación, con universitarios españoles de ciencias de la actividad física y el deporte, psicopedagogía, o ingeniería $\mathrm{y}$, fundamentalmente, con estudiantes de la rama de salud (Casuso-Holgado, Moreno-Moralez, Labajos-Manzanares, \& Montero-Bancalero, 2017; Extremera, Duran, \& Rey, 2007; López-Alonso, 2011; López-Alonso et al., 2016; Martínez \& Salanova, 2003; Vizoso \& Arias, 2016) se ha visto la necesidad de revalidarlo en el contexto de universitarios con unas características especiales, a saber, prepararse como educadores docentes de niños con edades comprendidas entre los 3 y los 18 años, lo cual implica que estos estudiantes deban tener vocación y sentirse comprometidos con sus estudios por ser los responsables de la formación inicial de los hombres y mujeres del futuro más próximo. Por eso la importancia de aplicar un análisis instrumental previo y de esta manera garantizar la fiabilidad y validez de los datos que se puedan manejar con el objeto de ofrecer resultados con garantía de veracidad y no cometer el error de replicar la escala sin comprobar su fiabilidad.

Lloret-Segura, Ferreres-Traver, Hernández-Baeza y Tomás-Marco (2014), recomiendan la utilización secuencial del análisis factorial exploratorio (AFE) y análisis factorial confirmatorio (AFC). Siguiendo sus sugerencias se ha optado por comenzar con el AFE con el fin de identificar, tanto en número como en composición, los factores comunes, o variables latentes, necesario para explicar la varianza común de la totalidad de los ítems analizados. Seguidamente se realizó el AFC para confirmar la adecuación y revalidación de la escala usada en el presente estudio.

Se utilizaron cuatro programas estadísticos, SPSS.22, Factor.10.8.01, G*Power 3.1.9.2 y MPlus.7.3.

Se calculan estadísticos univariados para cada ítem de la escala (media, desviación típica, varianza, asimetría, curtosis e índice de homogeneidad corregida) y el gráfico de sedimentación con el fin de obtener una primera aproximación al número óptimo de factores recomendado; se aplica como cálculo de la fiabilidad el coeficiente alfa de Cronbach $(\alpha)$ para datos ordinales.

Se comprueba la dimensionalidad de la escala mediante un AFE. Para determinar el número de factores se utiliza el método de Implementación Óptima de Análisis Paralelos, propuesto por Timerman y Lorenzo-Seva (2011) y se llevan a cabo 10.000 remuestreos. El análisis factorial se realiza a partir de las correlaciones policóricas entre variables; para la factorización se utiliza el método mínimos cuadrados no ponderados y se recurre al método Promin de rotación (Ferrando \& Lorenzo-Seva, 2014; Lorrenzo-Seva, 1999).

El programa M-Plus.7.3, se usa para realizar un AFC replicando los parámetros estimados y reespecificando el modelo si fuera necesario.

En el estudio empírico se realizará un análisis multivariado de la varianza (MANOVA) utilizando el paquete estadístico SPSS.22 y el cálculo del tamaño del efecto ( $d$ de Cohen) con el programa G*Power 3.1.9.2.

\section{Resultados}

Se han calculado los valores descriptivos, -tendencia central y distribución-, de las variables que componen la escala. Con respecto a la Asimetría (Asim.) y Curtosis (Cur.), se ha observado que las 17 variables, utilizando el rango -2, 2 (Bandalos \& Finney, 2010; Muthén \& Kaplan, 1985, 1992;) presentaban una buena distribución. El índice de homogeneidad corregido también presenta valores aceptables, todos por encima de .500 . Ante estos resultados se ha procedido a realizar el AFE con la totalidad de los ítems.

El gráfico de sedimentación (figura 1) muestra definidos dos factores.

Para el AFE se ha utilizado el programa Factor.10.8.01. Se ha probado si los participantes proceden de poblaciones con la misma varianza y si se justifica una buena adecuación muestral. El estadístico de Bartlett [3475.6 (Df=136; $p=0.000010)$ ] y el test de Kaiser-Meyer-Olkin $(K M O)=.93$, revelan un buen ajuste de los datos para ser sometidos a análisis factorial, y teniendo en cuenta el carácter ordinal del registro de los datos (escala Likert de 1 a 5) la extracción de factores en el AFE conviene realizarla mediante la estimación robusta de mínimos cuadrados no ponderados (ULS) y de matrices policóricas.

Los resultados del AFE explican el $58 \%$ de la varianza total de los dos factores extraídos. El índice de ajuste comparado (CFI) ha sido de .99 , el índice de bondad de ajuste (GFI) también de .99, el criterio de información bayesiano (BIC) de 411.900 y la raíz cuadrática media de los residuales (RMSR) de .047. Los datos extraídos indican ajuste razonable de la estructura bidimensional para estos ítems (García-Cueto, Gayo-Álvaro, \& Miranda-García, 1998).

Los resultados de la rotación (Promin) revelan que dos variables cargan en los dos factores a la vez con una diferencia en los valores inferior a .100 (V2 y V5).

Se ha tomado la decisión de prescindir de las dos variables que cargan en dos factores simultáneamente, la V2 y la V5, y el cuestionario ha quedado configurado con 15 variables distribuidas en dos factores, el F1: Predisposición al estudio (al que se conocerá como Pr.Es) y el factor F2: Satisfacción por el estudio (Sa.Es desde ahora). Los datos de la rotación sin las dos variables omitidas, la Comunalidad (Com.), el Índice de Homogeneidad corregido (IHc) y el Alfa de Cronbach pueden verse en la tabla 3.

Realizado el AFE, se ha procedido a comprobar los resultados mediante un AFC, utilizando para ello el paquete estadístico MPlus.7 tomado como referencia válida los valores de CFI y $T L I \geq .9$, RMRSA $\leq .08$ y $S R M R \leq .05$. Los resultados han mostrado que la variable 11 carga en la 10 y la variable 13 en 
Tabla 1 Medidas de tendencia central y dispersión de las variables del cuestionario UWES-S

\begin{tabular}{|c|c|c|c|c|c|c|c|}
\hline & M & Md & DT & Var. & Asim. & Curt. & IHc \\
\hline V1. En mis estudios se presentan nuevos retos & 4.05 & 4.00 & .932 & .869 & -.998 & .818 & .519 \\
\hline V2. En mis estudios me siento lleno/a de energía & 3.68 & 4.00 & 1.007 & 1.015 & -.670 & .117 & .691 \\
\hline $\begin{array}{l}\text { V3. Estoy inmerso/a y concentrado/a en mis } \\
\text { estudios }\end{array}$ & 3.86 & 4.00 & .876 & .767 & -.709 & .660 & .657 \\
\hline V4. Soy persistente en mis estudios & 4.01 & 4.00 & .942 & .887 & -.768 & .264 & .538 \\
\hline V5. Estoy entusiasmado/a con mis estudios & 4.00 & 4.00 & 1.020 & 1.040 & -.983 & .507 & .777 \\
\hline $\begin{array}{l}\text { V6. Puedo continuar trabajando en mis estudios } \\
\text { durante largos períodos de tiempo }\end{array}$ & 3.79 & 4.00 & .961 & .924 & -.666 & .323 & .599 \\
\hline $\begin{array}{l}\text { V7. Cuando me levanto por las mañanas tengo } \\
\text { ganas de ir a la Facultad }\end{array}$ & 3.02 & 3.00 & 1.095 & 1.198 & -.228 & -.585 & .652 \\
\hline $\begin{array}{l}\text { V8. Incluso cuando las cosas no van bien, continúo } \\
\text { estudiando }\end{array}$ & 3.65 & 4.00 & 1.030 & 1.060 & -.694 & .016 & .552 \\
\hline V9. Soy fuerte y enérgico/a en mis estudios & 3.79 & 4.00 & .896 & .803 & -.668 & .413 & .669 \\
\hline $\begin{array}{l}\text { V10. Aprendo cosas nuevas e interesantes en } \\
\text { mis estudios }\end{array}$ & 3.91 & 4.00 & 1.049 & 1.101 & -.906 & .286 & .678 \\
\hline V11. Mis estudios tienen sentido & 3.97 & 4.00 & 1.051 & 1.104 & -.895 & .166 & .659 \\
\hline $\begin{array}{l}\text { V12. Cuando estoy estudiando olvido todo lo que } \\
\text { pasa a mi alrededor }\end{array}$ & 2.92 & 3.00 & 1.047 & 1.097 & -.075 & -.728 & .511 \\
\hline V13. Me «dejo llevar» por mis estudios & 3.07 & 3.00 & 1.022 & 1.044 & -.323 & -.480 & .664 \\
\hline V14. Mis estudios son estimulantes e inspiradores & 3.43 & 4.00 & 1.092 & 1.191 & -.471 & -.334 & .748 \\
\hline V15. Estoy orgulloso/a de mis estudios & 4.25 & 4.00 & .926 & .857 & -1.365 & 1.758 & .600 \\
\hline $\begin{array}{l}\text { V16. Cuando estoy absorto/a en mis estudios, } \\
\text { me siento bien }\end{array}$ & 3.67 & 4.00 & .953 & .909 & -.522 & .065 & .640 \\
\hline V17. El tiempo vuela cuando estoy trabajando & 4.03 & 4.00 & 1.008 & 1.015 & -1.061 & .872 & .566 \\
\hline
\end{tabular}

Nota: M=Media; Md=Mediana; DT=Desviación Típica; Var.=Varianza; Asim.=Asimetría; Cur.=Curtosis; IHc=Índice de homogeneidad corregido

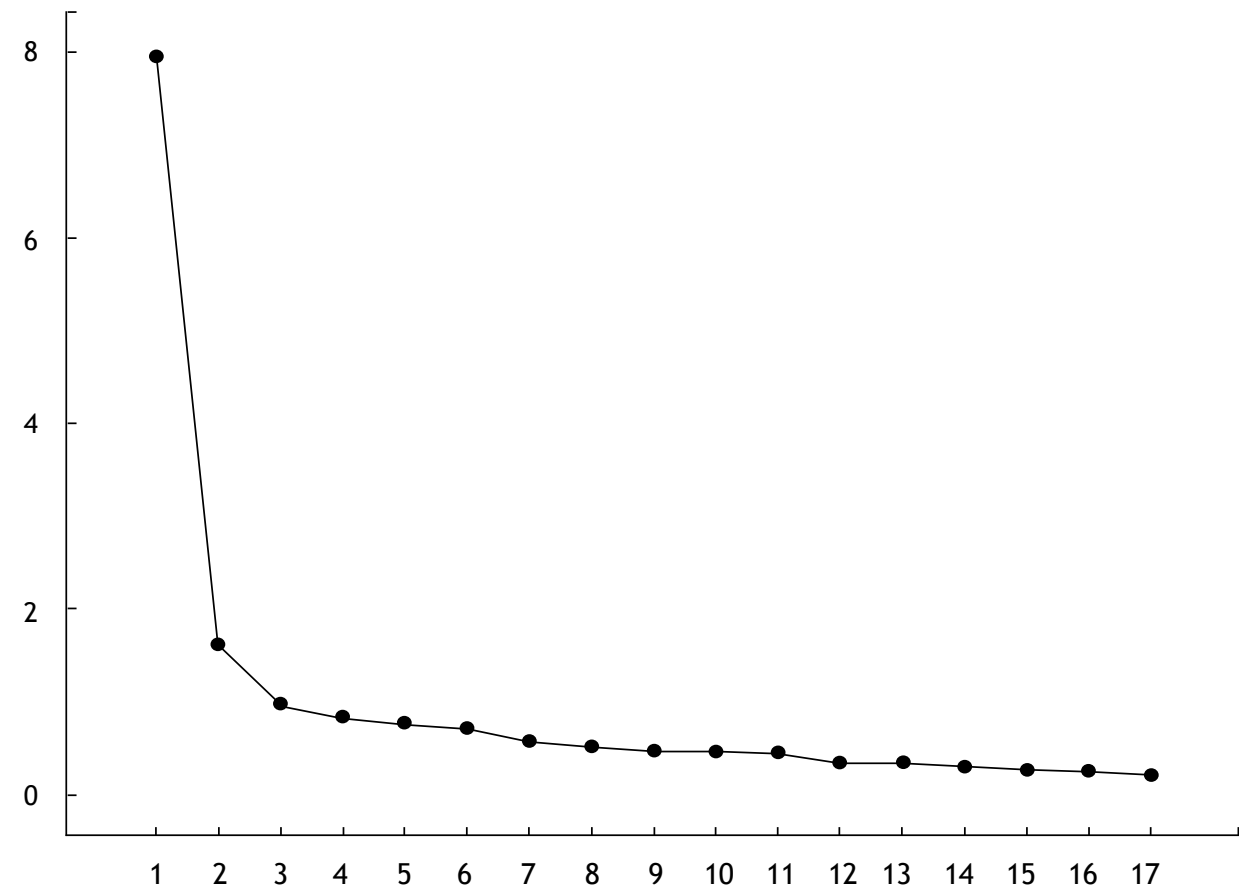

Figura 1. Gráfico de sedimentación del cuestionario UWES-S 
Tabla 2 Matriz de carga rotada y comunalidad

\begin{tabular}{|c|c|c|c|}
\hline \multirow{2}{*}{ Variables } & \multicolumn{2}{|c|}{ Matriz de carga rotada } & \multirow[b]{2}{*}{ Comunalidad } \\
\hline & $\mathrm{F} 1$ & $\mathrm{~F} 2$ & \\
\hline V 01. En mis estudios se presentan nuevos retos & -.077 & .681 & .390 \\
\hline V 02. En mis estudios me siento lleno/a de energía & .380 & .400 & .536 \\
\hline V 03. Estoy inmerso/a y concentrado/a en mis estudios & .791 & -.039 & .580 \\
\hline V 04. Soy persistente en mis estudios & .911 & -.247 & .550 \\
\hline V 05. Estoy entusiasmado/a con mis estudios & .461 & .429 & .696 \\
\hline $\begin{array}{l}\text { V 06. Puedo continuar trabajando en mis estudios durante largos períodos } \\
\text { de tiempo }\end{array}$ & .503 & .184 & .427 \\
\hline V 07. Cuando me levanto por las mañanas tengo ganas de ir a la Facultad & .480 & .236 & .458 \\
\hline V 08. Incluso cuando las cosas no van bien, continúo estudiando & .848 & -.200 & .502 \\
\hline V 09. Soy fuerte y enérgico/a en mis estudios & .917 & -.136 & .671 \\
\hline V 10. Aprendo cosas nuevas e interesantes en mis estudios & -.288 & 1.066 & .754 \\
\hline V 11. Mis estudios tienen sentido & -.307 & 1.088 & .773 \\
\hline V 12. Cuando estoy estudiando olvido todo lo que pasa a mi alrededor & .463 & .090 & .285 \\
\hline V 13. Me «dejo llevar» por mis estudios & .524 & .206 & .482 \\
\hline V 14. Mis estudios son estimulantes e inspiradores & .063 & .770 & .670 \\
\hline V 15. Estoy orgulloso/a de mis estudios & .145 & .560 & .457 \\
\hline V 16. Cuando estoy absorto/a en mis estudios, me siento bien & .255 & .451 & .442 \\
\hline V 17. El tiempo vuela cuando estoy trabajando & .085 & .544 & .373 \\
\hline
\end{tabular}

la 12 , motivo por el cual se ha procedido a la reespecificación del modelo obteniendo resultados favorables (tabla 4).

Comprobado el ajuste del cuestionario y definidos los factores, se ejecuta el estadístico de Levene para conocer si la homogeneidad de las varianzas se satisface, obteniendo buenos resultados para realizar análisis de varianza ( $p>.05)$, dato que indica que grupos son homogéneos (Ver tabla 5).

Antes de proceder al análisis de varianza se ha agrupado a los sujetos en tres grupos de edad, el primero con los que tienen menos de 21 años, el segundo entre 21 y 24 y el tercero los que tienen más de 24 años.

Se ha aplicado un análisis multivariado de la varianza, MANOVA $(2 \times 3)$ para determinar si la interacción entre las variables independientes, sexo y grupos de edad, afecta de manera conjunta sobre los dos factores estudiados.

Los resultados muestran un efecto de interacciones nulo, pero sí revelan diferencias estadísticamente indicadoras en función del sexo $\left(\lambda=.965, F_{(2,366)}=6.629, p \leq .001\right.$, $\left.\eta^{2}=.035,1-\beta=.911\right)$ y de las edades $\left(\lambda=.968, \mathrm{~F}_{(4,732)}=3.037\right.$, $\mathrm{p} \leq .05, \eta^{2}=.01,1-\beta=.805$.

Los análisis univariados revelaron diferencias estadísticamente significativas en cuanto al sexo en el factor Sa. Es $\left(F_{(1,372)}=10.896, D M=312, d=.44, p \leq .001\right)$ y en el factor Pr.Es $\left(\mathrm{F}_{(1,372)}=11.209, \mathrm{DM}=.339, d=.38, p \leq .001\right)$. La variable edad, no presenta diferencias estadísticamente indicativas (Tabla 6).
Se ha aplicado un MANOVA $(3 \times 3)$ para determinar la interacción entre las variables independientes, modalidad de estudios y universidad en la que trabajan. Los resultados presentan interacción nula, pero señalan diferencias estadísticamente significativas en cuanto a la universidad a la que pertenecen $\left(\lambda .962, \mathrm{~F}_{(4.728)}=3.566, p=.05, \eta \underline{2}=.019,1-\beta=.871\right)$.

Los análisis univariados indican diferencias estadísticamente explicativas relativas a la universidad a la que pertenecen, solamente Sa.Es $\left(\mathrm{F}_{(2.372)}=7.133, p \leq .001\right)$.

Se ha procedido a la aplicación de la prueba post hoc de Bonferroni para conocer las diferencias de las medias (DM), además de calcular el tamaño del efecto mediante la aplicación de la $d$ de Cohen $(d)$.

En el factor Sa.Es, los que cursan estudios en una universidad del norte de España obtienen una media significativamente más baja que los que los cursan en la universidad del sur $(D M=.300, p<.05$ y $d=.37)$ y también más baja que los que estudian en la universidad chilena ( $D M=.701, p<.05$ y $d=.85)$. Al estudiar dichas diferencias en el factor Pr.Es, los resultados indican que también en este factor los estudiantes de la universidad del norte puntúan estadísticamente significativo más bajo que los de la universidad del sur $(D M=.204, p<.05$ y $d=.35)$. En el resto de comparaciones por pares no han aparecido datos estadísticamente significativos. Ver tabla 6. 
Tabla 3 Matriz de carga rotada omitidas las cargas inferiores a .300 y las variables que cargan en dos factores, la Comunalidad y el Índice de Homogeneidad corregido y el Alfa de Cronbach de cada factor

\begin{tabular}{|c|c|c|c|c|}
\hline \multirow[t]{2}{*}{ Variables } & \multicolumn{2}{|c|}{$\begin{array}{c}\text { Matriz de carga rotada } \\
\text { Omitidas las cargas inferiores a } .300 \\
\end{array}$} & \multirow[b]{2}{*}{ Com. } & \multirow[b]{2}{*}{$\mathrm{IHC}$} \\
\hline & F1: Pr.Es & F2: Sa.Es & & \\
\hline V 09. Soy fuerte y enérgico/a en mis estudios & .865 & & .402 & .713 \\
\hline V 04. Soy persistente en mis estudios & .852 & & .731 & .618 \\
\hline $\begin{array}{l}\text { V 08. Incluso cuando las cosas no van bien, continúo } \\
\text { estudiando }\end{array}$ & .804 & & .671 & .628 \\
\hline V 03. Estoy inmerso/a y concentrado/a en mis estudios & .691 & & .749 & .673 \\
\hline V 13. Me «dejo llevar» por mis estudios & .534 & & .369 & .649 \\
\hline $\begin{array}{l}\text { V 12. Cuando estoy estudiando olvido todo lo que pasa } \\
\text { a mi alrededor }\end{array}$ & .463 & & .453 & .520 \\
\hline $\begin{array}{l}\text { V 06. Puedo continuar trabajando en mis estudios } \\
\text { durante largos períodos de tiempo }\end{array}$ & .462 & & .307 & .564 \\
\hline $\begin{array}{l}\text { V 07. Cuando me levanto por las mañanas tengo ganas } \\
\text { de ir a la Facultad }\end{array}$ & .451 & & .496 & .611 \\
\hline V 11. Mis estudios tienen sentido & & .986 & .523 & .785 \\
\hline $\begin{array}{l}\text { V 10. Aprendo cosas nuevas e interesantes en } \\
\text { mis estudios }\end{array}$ & & .984 & .516 & .748 \\
\hline V 14. Mis estudios son estimulantes e inspiradores & & .716 & .382 & .753 \\
\hline V 01. En mis estudios se presentan nuevos retos & & .605 & .342 & .544 \\
\hline V 17. El tiempo vuela cuando estoy trabajando & & .514 & .642 & .607 \\
\hline V 15. Estoy orgulloso/a de mis estudios & & .466 & .441 & .584 \\
\hline $\begin{array}{l}\text { V 16. Cuando estoy absorto/a en mis estudios, } \\
\text { me siento bien }\end{array}$ & & .431 & .504 & .572 \\
\hline $\begin{array}{l}\text { Coeficiente de fiabilidad } \\
\alpha \text { de Cronbach }\end{array}$ & .876 & .867 & & \\
\hline
\end{tabular}

Tabla 4 Valores de los índices de ajuste en los análisis factoriales confirmatorio

\begin{tabular}{lcccccc}
\hline & $\chi^{2}$ & $p$ & RMRSA & CFI & TLI & SRMR \\
\hline Valoración de los índices de ajuste del modelo original & 348.350 & .00 & .08 & .886 & .865 & .06 \\
Valoración de los índices de ajuste del modelo reespecificado & 270.207 & .00 & .07 & .919 & .902 & .05 \\
\hline
\end{tabular}

Tabla 5 Homogeneidad de varianzas

\begin{tabular}{lcc} 
& Estadístico de Levene & $p$ \\
\hline Satisfacción con los estudios & $1.783_{(369,2)}$ & .170 \\
Predisposición al estudio & $1.555_{(369,2)}$ & $.213^{(3)}$ \\
\hline
\end{tabular}

\section{Discusión}

La estructura factorial de la escala de Compromiso con los estudios de los futuros docentes El, EP y ES, ha sido el eje central del presente estudio. El cálculo se ha hecho mediante un análisis factorial que, con suficiente apoyo empírico, ha ofrecido una distribución bifactorial de la escala, F1: Pre- disposición a estudiar; F2: Satisfacción con el estudio. Este resultado difiere con el mostrado por Schaufeli y Bakker (2003), creador de Utrecht Work Engagement Scale (UWES), que confirmaba tres factores, Vigor, Absorción y Dedicación. El factor Predisposición a estudiar se corresponde con los que el autor de la escala denominó Vigor y Dedicación, concretamente con actitudes comportamentales e involucración 
Tabla 6 Comparación por pares y tamaño del efecto

\begin{tabular}{|c|c|c|c|c|}
\hline Factores & Pares & DM & $p$ & $d$ \\
\hline Satisfacción con estudios & Varón ${ }^{(1)}$ - Mujer ${ }^{(2)}$ & $.312^{(2)}$ & .001 & .44 (Medio) \\
\hline Predisposición al estudio & Varón' ${ }^{(1)}-$ Mujer $^{(2)}$ & $.297^{(2)}$ & .001 & .38 (Bajo) \\
\hline \multirow{2}{*}{ Satisfacción con estudios } & U.Sur(1) - U.Norte ${ }^{(2)}$ & $.300^{(1)}$ & .005 & .37 (Bajo) \\
\hline & U.Norte ${ }^{(2)}$ - U.Latinamer ${ }^{(3)}$ & $.701^{(3)}$ & .05 & .85 (Alto) \\
\hline Predisposición al estudio & U.Sur ${ }^{(1)}$ - U.Norte ${ }^{(2)}$ & $.204^{(1)}$ & .05 & .24 (Bajo) \\
\hline
\end{tabular}

de los futuros docentes en sus estudios, y el factor Satisfacción con los estudios puede semejarse al de Absorción, o la valoración de los estudios que cursan. Este resultado ratifica el obtenido por Parra y Pérez (2010), con estudiantes de psicología de dos universidades chilenas, a quienes aplicó el mismo cuestionario resumido en nueve ítems, que también concluyó con dos factores.

Las mujeres presentaron mayores puntuaciones en compromiso con los estudios en la satisfacción y en la predisposición que manifiestan, revalidando los resultados obtenidos en estudios anteriores (Cachón, Cuervo, Zagalaz, \& González, 2015; Extremera et al., 2007; Grau, Agut, Martínez, \& Salanova, 2000; Martínez \& Salanova, 2003), que manifiestan que las mujeres puntúan más altas en las subescalas de compromiso, frente a otros autores que no señalan distinciones por género (Benevides-Pereira, Fraiz de Camargo, \& Porto-Martins, 2009).

Un dato que no puede pasar desapercibido es la diferencia en la valoración del compromiso con los estudios según la zona geográfica dónde está ubicada la universidad. Los estudiantes del norte de España muestran valores significativamente más bajos que los del sur en ambos factores. Los mismos estudiantes muestran diferencias con los de la universidad chilena en el factor Predisposición del estudio, explicando, en este caso, un tamaño del efecto bastante alto. Por ello, es necesario continuar investigando este resultado replicando el estudio en otras universidades para comprobar si este dato se repite en cuanto a la situación geográfica, o solo es debido a que han sido pocas las universidades estudiadas.

\section{Agradecimientos}

Al programa de Doctorado en Innovación Didáctica y Formación del Profesorado de la UJA, que hace posible los trabajos de investigación conjuntos entre Universidades. A los Grupos de investigación HUM653, Innovación didáctica en actividad física (IDAF) del PAIDI y la UJA y Educación, Actividad Física, Deporte y Salud (EDAFIDES) de la UNIOVI, que han respaldado este trabajo. A los investigadores noveles que se han unido a esta investigación.

Este manuscrito cumple las normas éticas internacionales (American Psychological Association - APA y la Declaración de Helsinki) y con la legislación española requerida para este tipo de investigación.

\section{Referencias}

Arraigada, M. \& Musticchio, A. (2015). Estudio sobre la relación de los docentes universitarios con su trabajo desde la Salud Ocupacional Positiva. XIX Jornadas de Docentes e Investigadores de recursos humanos de la Argentina y $X$ del Conosur. Temuco, Argentina.

Bandalos, D.L., \& Finney, S.J. (2010). Factor analysis: Exploratory and confirmatory. En G. R. Hancock y R. O. Mueller (Eds.), Reviewer's guide to quantitative methods. New York, NY: Routledge.

Belando, N., Ferriz-Morell, R., \& Moreno-Murcia, J.A. (2012). Propuesta de un modelo para la mejora personal y social a través de la promoción de la responsabilidad en la actividad físico-deportiva. RICYDE. Revista internacional de ciencias del Deporte, 29(8), 202-222. http://dx.doi.org/10.5232/ricyde2012.02902.

Benevides-Pereira, A.M.T., Fraiz de Camargo, D., \& Porto-Martins, P.C. (2009). Utrecht work engagement scale, manual en español. Recuperado de http://www.schaufeli.com/downloads/ tests/Test\%20manual\%20UWES\%20Espanol.pdf.

Cachón, J., Cuervo, C., Zagalaz, M.L., \& González, C. (2015). Relación entre la práctica deportiva y las dimensiones del autoconcepto en función del género y la especialidad que cursan los estudiantes de los Grados de Magisterio. Jour Sport and Health Research, 7(3), 257-266.

Carrasco, A.M., Corte, C.M., \& León, J.M. (2010). Engagement: un recurso para optimizar la salud psicosocial en las organizaciones y prevenir el burnout y estrés laboral. Revista Digital de Salud y Seguridad en el Trabajo, 1, 1-22.

Casuso-Holgado, M.J. (2011). Estudio del estrés, engagement y rendimiento académico en estudiantes universitarios de ciencias de la salud (Tesis doctoral). Universidad de Málaga, España.

Casuso-Holgado, M.J., Moreno-Morales, N., Labajos-Manzanares, M.T., Montero-Bancalero, F.J. (2017). Características psicométricas de la versión española de la escala UWES-S en estudiantes universitarios de Fisioterapia. Fisioterapia 39(1), 39:4-9 http://dx.doi.org/10.1016/j.ft.2016.02.003

Chen, X., Maoa, Y., Kong, L., Li, G., Xin, M., Lou, F., \& Li, P. (2016). Resilience moderates the association between stigma and psychological distress among family caregivers of patients with schizophrenia. Personality and Individual Differences, 96, 78-82.

Da Silva-Cardoso, A.M., León-Jariego, J.C., Perea-García, M.C., \& Bermejo-Contioso, I. (2014). Convencionalidad social y engagement en estudiantes universitarios ¿La conformidad social fomenta el engagement en los estudiantes universitarios? Atas do I Congresso Internacional Envolvimento dos Alunos na Escola: Perspetivas da Psicologia e Educação. Instituto de Educação da Universidade de Lisboa, Portugal.

Extremera, N., Durán, A., \& Rey, L. (2007). Inteligencia emocional y su relación con los niveles de burnout, engagement y estrés en estudiantes universitarios. Revista de Educación, 342, 239-256. 
Ferrando, P.J. \& Lorenzo-Seva, U. (2014). El análisis factorial exploratorio de los ítems: algunas consideraciones adicionales. Anales Psicología, 30(3), 1170-1175. http://dx.doi.org/10.1016/ j.ft.2016.02.003.

García, F. \& Musitu, G. (2014). Manual AF-5 Autoconcepto. Forma 5. Barcelona, España: TEA.

García-Cueto, E., Gayo-Álvaro, P., \& Miranda-García, R. (1998). Bondad de ajuste en el análisis factorial confirmatorio. Psicothema, 10(3), 717-724.

Gómez, P., Pérez, Cr., Parra, P., Ortiz, L., Matus, O., McColl, P., Torres, G., Meyer, A. (2015). Relación entre el bienestar y el rendimiento académico en alumnos de primer año de medicina. Revista Médica de Chile, 143, 930-937.

Grau, R., Agut, S., Martínez, I., \& Salanova, M. (2002). Safety attitudes and its relationship with safety training and generalized self-efficacy. Internacional Journal Occupational Safety and Ergonomics, 8(1), 23-35. http://dx.doi.org/10.1080/10803548. 2002.11076512

Lloret-Segura, S., Ferreres-Traver, A., Hernández-Baeza, A., \& Tomás-Marco, I. (2014). El análisis factorial exploratorio de los ítems: una guía práctica, revisada y actualizada. Anales de Psicología 30(3), 11511169. http://dx.doi.org/10.6018/analesps.30.3.199361

López-Alonso, A. I. (2011). Enfoques de aprendizaje de los alumnos universitarios. Interrelación con hábitos de ocio, engagement y percepción de bienestar. Propuesta de un modelo estructural (Tesis doctoral). Universidad de León, España.

López-Alonso, A.I., López-Aguado, M., Fernández-Martínez, M.E., Liébana Presa, Cr., \& Gutiérrez-Provecho, L. (2016). Los enfoques de aprendizaje, el engagement, el ocio y el rendimiento anterior. Propuesta de un modelo. Bordón, 68(4), 67-88. http://dx.doi.org/10.13042/Bordon.2016.40940

Lorenzo-Seva, U. (1999). Promin: a method for oblique factor rotation. Multivariate Behavioral Research, 34, 347-356.

Lykken, D.T. (2000). Las personalidades antisociales. Barcelona, España: Herder.

Martínez, I.M., \& Salanova, M. (2003). Niveles de burnout y engagement en estudiantes universitarios. Relación con el desempeño y desarrollo profesional. Revista de Educación, 330, 361-384.

Maslach, C. \& Leiter, M.P. (1997). The truth about burnout. New York, NY: Jossey-Bass.

Mesana, M.I. (2013). Alimentación en adolescentes: Valoración del consumo de alimentos y nutrientes en España: Estudio Avena (Tesis Doctoral). Universidad de Zaragoza, España.

Muthén, B., \& Kaplan, D. (1985). A comparison of some methodologies for the factor analysis of non-normal Likert variables. British Journal of Mathematical and Statistical Psychology, 38, 171-189.

Muthén, B. \& Kaplan D. (1992). A comparison of some methodologies for the factor analysis of non-normal Likert variables: A note on the size of the mode, British Journal of Mathematical and Statistical Psychology, 45, 19-30.

Organización Mundial de la Salud (2004). Programa Conjunto de las Naciones Unidas sobre el VIH/sida y Fondo de Población de las Naciones Unidas. Seen but Not Heard: Very young adolescents aged 10-14 years, 5-7. ONUSIDA, Ginebra, Suiza.
Paniagua, G. (2009). Monográfico: El desarrollo de la El: un crecimiento costoso. CEE Participación Educativa, 12, 20-34.

Parra, P., \& Pérez, C. (2010). Propiedades psicométricas de la escala de compromiso académico, UWES-S (versión abreviada), en estudiantes de psicología. Revista de Educación y Ciencias de la Salud, 7(2), 128-133.

Parra, P. (2014). Relación entre el nivel de engagement y el rendimiento académico teórico/práctico. Revista de Educación y Ciencias de la Salud, 7(1), 57-63.

Ponce, F. P. (2015). Análisis exploratorio de modelos de ecuaciones estructurales sobre la escala de resiliencia de Connor y Davidson (CD-RISC) en Chile y España. Salud \& Sociedad, 6(3), 238-247.

Raigosa, D., \& Marín, B. (2010). Formación en creencias de eficacia. Una propuesta para reducir el burnout y optimizar los niveles de engagement en empleados. International Journal of Psychological Research, 3(2), 86-92.

Real Academia de la Lengua Española (2016). Diccionario de la RAE (22 ${ }^{\mathrm{a}}$. ed.). Madrid, España.

Rosales, C. (2014). ¿Cómo será mi profesión de maestro? Tendencias Pedagógicas, 23, 29-44.

Salanova, M., \& Schaufeli, W.B. (2004). El engagement de los empleados: un reto emergente para la dirección de los recursos humanos. Estudios Financieros, 261, 109-138.

Schaufeli, W. B., Martínez, I. M., Marqués-Pinto, A., Salanova, M., \& Bakker, A.B. (2002). Burnout and engagement in university students: A cross-national study. Jour Cross-Cultural Psychology, 33(5), 464-481.

Schaufeli, W.B., \& Bakker, A.B. (2003). Original: UWES - Utrecht Work Engagement Scale preliminary manual. Occupational Health Psychology Unit. Utrecht University

Shavelson, R.L., Hubner, J.L., \& Stanton, G.C. (1976). Self concept validation of construct integrations. Review of Educational Research, 46, 407-441.

Silva, Y.F., Carena, M.Y., \& Canuto, M.F. (2013). Niveles de engagement y burnout en voluntarios universitarios Un estudio exploratorio y descriptivo. Boletín de Psicología, 108, 37-57.

Timerman, M.E., \& Lorenzo-Seva, U. (2011). Dimensionality assessment of ordered polytomous items with parallel analysis. Psychological Methods, 16(2), 209-20. http://dx.doi.org/10.1037/ a0023353

Villarreal, M.E., Sánchez, J.C., \& Musitu, G. (2010). Como mejorar tus Habilidades Sociales. Programa para adolescentes en manejo del estrés, resolución de problemas, autoestima, asertividad, proyecto de vida y administración del tiempo libre. Universidad Autónoma de Nuevo León, Monterrey/Universidad Pablo de Olavide de Sevilla.

Vizoso, C.M., \& Arias, O. (2016). Estresores académicos percibidos por estudiantes universitarios y su relación con el burnout y el rendimiento académicos. Anuario de Psicología 46(2), 90-97.

Zagalaz, M.L., Cachón, J., \& Lara, A.J. (2014). Fundamentos de la programación de Educación Física en primaria. Síntesis. Madrid, España. 